\title{
The Development of an MIS for Iron Ore Mining Operations
}

\author{
J.E. Everett \\ The University of Western \\ Australia \\ jeverett@ecel.uwa.edu.au
}

\author{
T. Howard \\ BHP Iron Ore
}

\section{Kamperman BHP Iron Ore}

\begin{abstract}
We describe the development and implementation of a management informing system (MIS) to aid the mining, transporting, stockpiling and shipping of iron ore. Ore is railed from mines to a port, where it is blended on stockpiles and recovered to ships. The project commenced as a Decision Support System to aid quality control of ore grade. It evolved to become an MIS aiding operators, decision makers and managers at multiple stages and levels of the production process. The project's history provides valuable lessons for the development of an MIS. The project was designed and implemented with clients rather than for clients, ownership by the users was emphasized, and domain knowledge was sought and incorporated at every stage. Contrary to textbook prescription, these considerations suggest that, rather than basing a project upon some initial grand plan, an incremental evolution is preferable, with developers and users cooperatively exploring possibilities as they unfold.
\end{abstract}

Keywords: MIS, DSS, Mining, Simulation, Excel.

\section{Introduction}

This paper describes the development and implementation of a Management Informing System (MIS) to aid the sequence of operations involved in mining, transporting, stockpiling and shipping of iron ore.

The mining and export of iron ore is of considerable economic importance to Australia, which produces over 150 million tonnes each year. In 1998-99, iron ore exports yielded Aus $\$ 3.8$ billion, or 5\% of the country's total merchandise exports (Australian Bureau of Statistics, 2001).

Iron ore is extracted at inland mines in Western Australia and railed to Port Hedland. At the port, the ore is crushed, assayed, and stacked onto stockpiles. The stockpiles are then reclaimed and the ore is loaded on to ships. Customers, mainly overseas, use the ore as input to blast furnaces. Successful operation of a blast furnace requires that suc-

Material published as part of this proceedings, either on-line or in print, is copyrighted by the author with permission granted to the publisher of Informing Science for this printing. Permission to make digital or paper copy of part or all of these works for personal or classroom use is granted without fee provided that the copies are not made or distributed for profit or commercial advantage AND that copies 1) bear this notice in full and 2) give the full citation on the first page. It is permissible to abstract these works so long as credit is given. To copy in all other cases or to republish or to post on a server or to redistribute to lists requires specific permission from the author. cessive inputs of iron ore have consistent composition, not only in iron, but also in several other minerals, particularly in phosphorus, silica and alumina. Suppliers are judged by their product's conformity to an agreed composition (TEX, 1995).

The quality of iron ore therefore depends greatly upon the uniformity of composition. Sales contract renewal and price negotiations depend on this quality. A producer's competitive strength is therefore very dependent on shipping ore of consistent and uniform composition.

\section{Stress - a measure of product quality}

The quality objective is to load ships with ore that matches the target composition, not only in iron but also in alumina, silica and phosphorus.

Although a customer is more likely to complain about a shortfall in iron, or excess of the other minerals, deviations in the opposite direction represent an opportunity cost. So the objective function is multidimensional, and symmetric around the target composition.

For each mineral, a non-dimensional stress component can be defined by dividing the deviation from target by an appropriate tolerance. The tolerances were estimated by marketing staff, and represent equal customer annoyance 
for each mineral. The square root of the summed squared stress components defines an "Aggregate Stress".

The Aggregate Stress is used as a single objective function, to be minimized for optimum quality.

\section{Iron Ore Production}

The iron ore production system can be summarized as shown in Figure 1. It is convenient to divide the production sequence into four stages as shown. At each stage there is opportunity to control the ore quality.

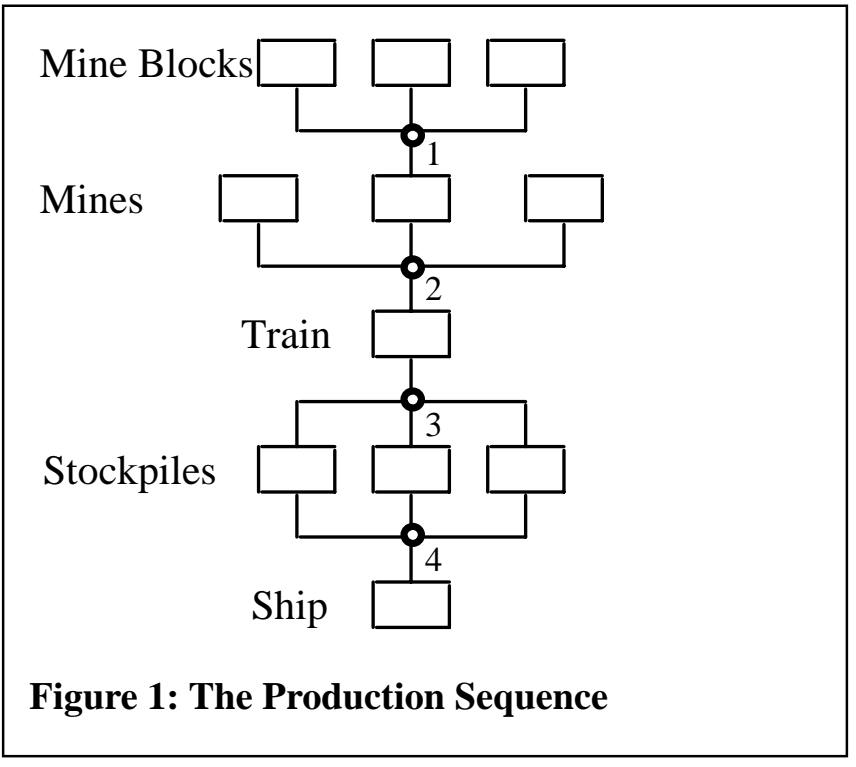

\section{Stage 1}

The iron ore mines are open-cut. The ore is extracted in steps 12 metres high. Each step is divided into areas, referred to as "blocks". A block is drilled with a pattern of vertical holes. The drill hole material is sampled and assayed to estimate the block's composition.

Unfortunately, the estimate of composition, from the drill hole material, is quite unreliable. As well as random error, there is systematic error because the lighter and finer minerals tend to blow away, causing silica and alumina to be underestimated and iron content to be overestimated.

The drill holes are filled with explosive and the ore is then blasted. At any one time there will therefore be a number of available blasted blocks, with estimated compositions.

At each shift, the Quality Controller decides how many truckloads (each of about 160 tonnes) are to be taken from which available blocks. This choice should be largely guided by the goal of producing ore of target composition, but must also influenced by operational cost and feasibility constraints.

\section{Stage 2}

The truckloads of ore are coarsely crushed and loaded through hoppers onto trains (of about 13 kilotonne capacity), which take the ore to the port.

A single ore product may be blended from multiple mines, either by trains being partly filled in more than one mine, or by railing trains in sequence from the different mines. The Train Controller is responsible for train sequencing, again with the purpose of delivering product of uniform and consistent composition.

\section{Stage 3}

When the ore trains arrive at the port they are further crushed, and then stacked onto stockpiles. Ore being conveyed from crusher to stacker is sampled and assayed. These port assays are considerably more accurate than the mine estimates, but unfortunately the information is not available until the ore has been stacked.

Usually, the crushed ore is divided into lump and fines components, which are stacked on separate stockpiles and marketed as different products.

In the operation to be considered in this paper, each stockpile is built to completion (typically 250 kilotonnes, or about 20 trainloads) before starting the next stockpile.

Studies for another operation have explored the improvement in composition consistency attainable by building multiple stockpiles in parallel. Everett $(1996,1997)$ discusses the improvement in composition consistency that was achieved.

\section{Stage 4}

Ships arrive at the port and are loaded. There is again the opportunity to increase composition uniformity by loading from multiple stockpiles. Everett $(1996,1997)$ discusses quality control at Stage 4 of the production sequence.

Stage 4 is not considered in this paper, but the work described here has led on to a project currently considering Stage 4 improvement. 


\section{An MIS for Iron Ore Mining Operations}

\section{The Continuous Stockpile Model}

The history of the project will be discussed in the next section. Although some features of the Continuous Stockpile Management System (CSMS) were developed during the project, it is convenient to begin by summarizing its salient features. A fuller description of the CSMS has been published (Everett \& Kamperman, 1999, Everett, 2001).

Previously, the objective had been to build each stockpile to target composition. However, this gave rise to excessively tight linkage between port and mine. Commonly, the mine would be asked to provide particular material to solve a problem at the port. After delays and natural variation, this special action often created new problems for the next stockpile instead of arriving in time to solve problems for the previous stockpile.

Improvement was attempted by aiming to maintain the composition of a moving average of the past few trains. This leads to bad decisions because an anomaly does not only cause a blip when it occurs, but also at an arbitrary delay when it leaves the moving average.

Ideally, new information should be given the most importance, and its importance should die away smoothly with time. This suggests that the composition should be exponentially smoothed, to give the composition of a Continuously Smoothed Stockpile (CSS). When mine blocks or trains are being selected, they are chosen so as to minimize the Aggregate Stress of the resulting CSS.

\section{Exponential Smoothing}

Exponential smoothing is used widely to summarize and forecast data (Hanke \& Reitsch, 1998). Usually the technique is applied to uniformly spaced data of equal importance.

In this application, successive amounts of material from mine blocks or trains revise the smoothed composition of the CSS.

Contributions from successive trains or mine blocks will have differing tonnages, and this must be considered in applying the exponential smoothing.

Consider a CSS with composition vector $\mathrm{C}_{1}$. Adding material of composition $\mathrm{S}$ and tonnage $\mathrm{t}$ results in a CSS whose composition vector $\mathrm{C}_{2}$ is given by:

$$
\mathrm{C}_{2}=\alpha \mathrm{S}+(1-\alpha) \mathrm{C}_{1}
$$

For consistency, the exponential smoothing constant $\alpha_{t}$ depends upon the tonnage t. Let $\alpha_{1}$ be the exponential smoothing constant per kilotonne. Everett (2001) shows that consistency requires:

$$
\alpha_{\mathrm{t}}=1-\left(1-\alpha_{1}\right)^{\mathrm{t}}
$$

\section{Choosing the smoothing constant}

The question remains as to how large the exponential smoothing constant per kilotonne should be. Simulation studies show that system performance is not sensitive to the choice of smoothing constant so long as it remains below 0.1 per kilotonne, as shown in the following graph (from Everett, 2001).

Since stockpiles are built to a capacity of about 250 kilotonnes, it seems intuitively reasonable that the smoothing constant should be of comparable magnitude.

A smoothing constant of 0.004 per kilotonne causes the influence of information to fall to $1 / \mathrm{e}$ of its original value by the time 250 kilotonnes of further material have been stacked. Accordingly this value of 0.004 per kilotonne for the smoothing constant was adopted for the work described here. (See Figure 2.)

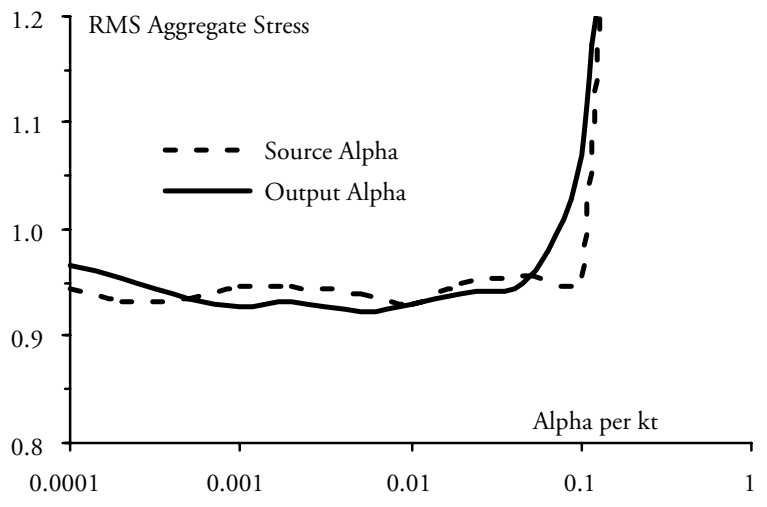

Figure 2: Choosing the Smoothing Constant

\section{History of the Project}

The MIS to be described here was not designed as a prespecified project, but was preceded by a series of technical and simulation studies, not initially aimed at the development of an MIS. The work evolved through the following phases. 


\section{Everett, Howard \& Kamperman}

\section{Early studies of stacking and ship loading}

A consultant carried out a series of technical and simulation studies in 1991-1992, to aid in the redesign of the Port Hedland ore handling and stockpiling facilities.

These studies suggested using the deviations from target divided by a tolerance as a non-dimensional stress measurement for each mineral. The component stress measurements could then be combined to form an objective function, "Aggregate Stress" (the square root of the summed squared component stresses), which would be used as a single non-dimensional composite measure of ore quality.

The studies also made extensive use of exponential smoothing, for forecasting and for summarizing the variations in composition.

The 1991-1992 studies were used in the engineering design and layout of the company's new port facilities. However, their implications were not followed through to influence the operational system.

Fortunately, the studies were well documented, and the reports were retained in the company's library.

Results from these studies and from a subsequent study of the port stacking and ship loading system for another company's operations have been published (Everett, 1996, 1997).

\section{Train control for Newman product}

In 1998, difficulties were being encountered in the operation of the Train Control system, controlling the train sequence from three mines in the Newman district.

Ore from the three mines, Whaleback, Jimblebar and Ore Body 25, is blended to form Newman product.

Whaleback is a rich deposit, and unique. When it runs out, production in the area will cease unless other deposits of similar quality have been discovered.

Jimblebar and Ore Body 25 are of lower grade, and several other similarly sub-economic ore bodies are available in the region.

Trains carrying iron ore are dispatched from the three mines in sequence. Arriving at Port Hedland, the trains are unloaded and the ore stacked onto a stockpile. Each stock- pile is built to completion. It is then available to be reclaimed for shipment, and the next stockpile begins to be built. The stockpiles are built and recovered in such a way that each can be assumed uniformly mixed.

The iron ore quality depends upon making sure that each stockpile is close to target composition, and this depends upon the appropriate sequencing of trains from the three mines.

Each mine has a different average composition, for each mineral. Successive trains from each mine differ in composition. Each day, about ten trains are dispatched. The train sequence has to be determined with no knowledge of the individual train's composition.

When the train is dispatched, a rough estimate of the train's composition becomes available. However, an accurate assay cannot be obtained until the ore has been crushed, after the train has been unloaded at port. The port assays are not available until after the ore has been stacked to a stockpile.

Until 1998, the composition of each stockpile was monitored while the stockpile was being built at the port. Port personnel communicated with the Train Controller. He then attempted to send trains in such a sequence that the stockpile was close to target composition on completion.

Many problems of coordination arose. A train designed to fix a problem at the end of one stockpile might be delayed and end up on the next stockpile. Bursts of effort were concentrated on composition control as each stockpile approached completion.

The over-tight coupling between port and mine production often led to problems of hunting, or over-compensation. Reaction to problems tended to rely on anecdotal evidence. The multiple objectives of target composition in each of several minerals were not combined. Generally, the most troublesome current objective would drive operation, and the other objectives would be ignored until they became troublesome.

The Train Controller read the 1991-1992 reports in the company archives, and decided that methods using exponential smoothing and Aggregate Stress could be used to decouple the port, train dispatch, and mining operations.

The Train Controller contacted the consultant who had written the 1991-1992 reports. They developed the concept 


\section{An MIS for Iron Ore Mining Operations}

of a Continuous Stockpile Management System (CSMS), using exponential smoothing, to decouple the port, train dispatch, and mining operations.

Instead of trying to complete each individual stockpile at target composition, the CSMS would try to maintain minimum Aggregate Stress for a continuous stockpile. For each train unloaded, the continuous stockpile's composition would be computed using exponential smoothing, with an alpha value per kilotonne equal to the reciprocal of the typical stockpile size.

To test the proposed methods, they designed and carried out a series of simulation studies, using data from the previous two years of production. The simulation model was constructed using Extend, an animated graphical user interface (GUI) package.

The simulation studies modeled the use of a CSMS and its effects on system performance. Exponentially smoothed compositions were maintained for the sequence of trains unloaded at port, and for the sequence of trains dispatched from each mine. These records enabled each successive train's mine source to be chosen so as to minimize the expected Aggregate Stress of the Continuous Stockpile.

The simulation studies showed that if a CSMS had been used over the previous two years, it would have halved the composition variability.

The studies also showed that twenty percent less tonnage of ore would have been extracted from Whaleback, the high-grade mine. A correspondingly greater tonnage would be extracted from the lower-grade mines, Jimblebar and Ore Body 25, which had lower operating cost. This suggested a potential twenty percent increase in the life of Whaleback (and of the mining community in the region), and considerable savings in operating cost.

Figure 3 shows some of the results from the simulation study, and throws light upon how the improvement was achieved. The graph shows the cumulative distribution of the composition in iron content for completed lump stockpiles, with the trains being sent in their actual sequence. This is with the cumulative distribution for trains being sent in the revised sequence so as to optimize the composition of the CSS.

Customers complain if the iron content is below specification, but not if it is above specification. Previously the variation in the composition of completed stockpiles was

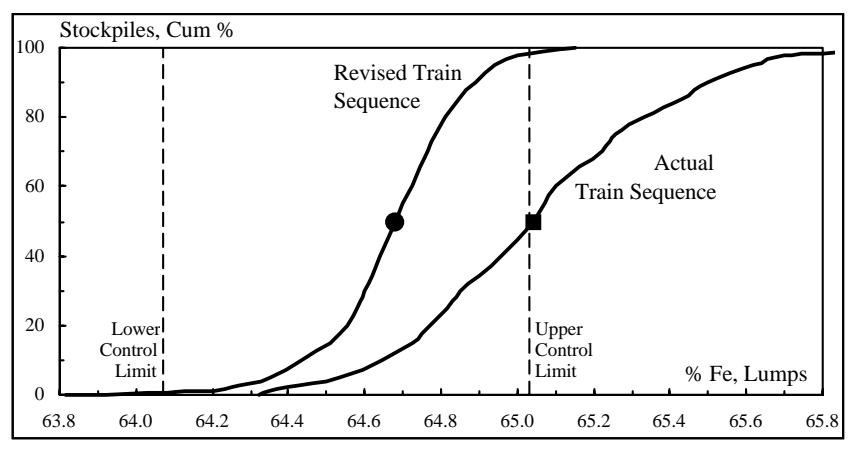

\section{Figure 3: Simulation Results}

much larger. Consequently, to avoid shipping product below specification, the actual sequence shipped product above specification.

The CSS provided greater control so that the simulated revised sequence avoided sending out product above specification, without increasing the danger of sending product below specification. This enabled a larger proportion of trains to be dispatched from the poorer quality mines, Jimblebar and Ore Body 25, conserving production from Whaleback, the valuable richer source.

Following review of the simulation studies, it was decided that a Decision Support System (DSS) should be developed to aid the Train Controller in selecting the sequence of trains from the three mines.

Under the previous system, the Train Controller and his colleagues had been using Excel spreadsheets to keep track of train compositions. It was realized that successful implementation of the CSMS would require more sophisticated data management, but that the chance of successful implementation would be enhanced by modeling the system in a way that operators were familiar with, and incorporating their domain knowledge.

The DSS was designed around an Excel workbook, with buttons to run Visual Basic macros. These macros were written to perform the various scheduling and data management operations. A good introduction to writing Visual Basic macros for Excel-based decision support systems is available in Albright (2001).

The advantage of this approach was that the operators were already familiar with using Excel. It was not a large step for them to become familiar with pressing buttons on the worksheets to operate invisible macros, to carry out the 


\section{Everett, Howard \& Kamperman}

data management operations, many of which they had previously done manually on similar-looking spreadsheets. There was also the advantage that the company already had Excel licensed on all its computers, so there were no problems of software compatibility or availability.

From this phase on, great care was taken to work with the operators. As each successive version of the DSS was developed, feedback was continually sought and acted upon. The operators had considerable domain knowledge, knowledge otherwise unavailable to the consultant, and they provided many suggestions.

Not only did this approach reduce embarrassing errors on the part of the consultant, but it also gave the operators a sense of ownership. From the beginning, it was very clear that the operators saw the system as something they wanted to work. This acceptance was helped by the fact that the operators were mining engineers who wanted the data maintenance tasks streamlined so that they could spend more time in the mine pit, monitoring operations. They continually suggested new potential applications for the system, and these suggestions were incorporated in successive versions of the DSS.

After six months operation, the DSS was reviewed, and it was confirmed that the benefits projected by the simulation studies were indeed being achieved.

\section{Mine and train control for Yandi product}

The company also mines iron ore at a nearby location called Yandi. The Yandi product is handled and marketed separately from the Newman product. Yandi comprises two pits, of different average composition. For each shift, train loads of a given tonnage must be sent from each pit, from selected mine blocks. It was attempted to select these mine blocks so as to control composition. Unfortunately, the composition of ore in each mine block can be only roughly estimated, from assays of material extracted while drilling the blast holes. These assays have not only random error, but also systematic bias because the lighter components of the ore tend to be blown away during drilling. These lighter components are lower in iron and higher in silica and alumina, so assays of the blast hole samples are biased.

Yandi's operation suffered from coordination and data problems similar to those described already for Newman. The company's Competitive Improvement Manager now joined the development team. It was decided to develop a
DSS for Yandi, to aid the choice of mine blocks from which to take each shift's production.

As before, the operators were heavily involved at every phase in the development of the DSS. The DSS again used Visual Basic macros embedded in an Excel workbook.

Decisions as to which mine blocks to use have to be based on the blast hole assays. These estimates are timely but inaccurate. Accurate assays are not available until after the ore has been railed to port, crushed and stacked to stockpile, too late to be used for decision making.

The two sets of assays were combined to obtain more timely and accurate information. This was done by maintaining exponentially smoothed records of blast hole estimates and of port assays for the mined ore. The current difference between these two smoothed compositions provided an adjustment, which was used to remove the bias error from the blast hole estimated assays. This bias correction was maintained separately for each of the two Yandi pits.

The Yandi DSS was introduced in late 1999, and has been found to improve the composition variability, and greatly to ease the computational burden for the operators. As a side benefit, the system provides reports for daily conference calls from Yandi to the management at Newman, and daily generates shift plans to instruct the mining contractors where to take ore.

The accumulated data on mine estimates and port assays has also enabled post-mortems to be carried out after operational glitches, greatly increasing the accountability and understanding of the system's operation.

\section{Optimization procedures}

Both the Newman and Yandi systems required the selection of material from available sources to optimize the quadratic Aggregate Stress objective function. This is actually a quadratic programming problem, which can be solved by a modified Simplex Algorithm (Taha, 1987).

However, instead of designing a quadratic programming solution, we used the Excel Add-In called Solver, driven by macros, to find an iterative solution whenever mine blocks or trains were being selected to optimize the CSS.

This procedure replaced the judgment-based decisions that had previously been made, resulting in considerable in- 


\section{An MIS for Iron Ore Mining Operations}

crease in performance and accountability, and requiring far less operator time.

\section{Further integration and development}

After the Decision Support Systems (DSS) were introduced to aid the Newman and Yandi operations, both systems were successively modified and enhanced, mainly at the request and suggestion of the operators. Many data maintenance functions previously carried out by hand or on individual Excel spreadsheets are now incorporated in the DSS.

The company's Mine Planners have also realized that the DSS, and its graphical outputs, is invaluable not only for helping day-to-day operations and reporting, but also in preparing monthly and yearly plans and reports.

Consider the four production stages shown in Figure 1.

The early 1991-1992 work comprised technical and simulation studies for Stage 3 and Stage 4, in the handling of ore from train to stockpile and stockpile to ship.

The simulation studies of 1998 examined the feasibility of using the Continuous Stockpile Management System to sequence the selection of trains from multiple mines, in Stage 2. As a result of these studies, a Decision Support System was developed for Stage 2. The Yandi Decision Support System applies the methods to Stage 1.

It is now planned to develop Decision Support Systems at the other mines (Stage 1) and at the port (Stage 3 and Stage $4)$.

\section{From DSS to MIS}

The systems described were initiated to provide decision support, specifically in the choice of source material to yield product of uniform composition. In this area of decision support, a computer-based system is clearly preferable to human judgment.

As the systems developed, more requirements were added. Some of these additional requirements related directly to decision support. For example, the Yandi operation developed from the original system, where all ore was crushed to a fines product, to a more sophisticated operation where some of the trainloads were separated into lumps and fines components. The decision support system (DSS) was accordingly modified to choose source material to optimize a weighted combination of the compositions of both products.

Once the system was established, it became clear to operators and managers that it could also be helpful as a Management Informing System (MIS). Over the past couple of years, MIS applications have continually been added to aid operational control and reporting and also to help both short-term and long-term planning. A few of the MIS developments will be outlined.

\section{Operational control}

The DSS is guided by estimates of composition of the potential sources at the mine. As described above, it adjusts the timely but inaccurate mine estimates, using the accurate port assays, that become available too late to be used for the material to which they directly relate. The adjusted mine estimates, with systematic error removed, improve the ability to choose source material to match the required composition.

Integration with the company's existing database systems has replaced the earlier need for data to be manually reentered. This has reduced the previous loss of time, operator burden, and chance of error.

Each day, the mining contractor's are given a plan, instructing them how many truckloads to take from where in the mine, and in what sequence. This daily plan is now generated automatically by the system and transmitted electronically to the contractor. At the end of each shift, the contractor electronically returns a report on the material mined. This procedure greatly facilitates reconciliation between plan and performance, and thus improves operational control.

\section{Reporting}

The Yandi mine is physically remote from the mines around Newman, and both sites are several hundred kilometers from Port Hedland.

Each morning, operators and managers at all three sites gather for a telephone conference call. Before the conference call, performance reports generated by the DSS/MSS are distributed by e-mail, greatly enhancing the conference call procedure. 


\section{Everett, Howard \& Kamperman}

\section{Short-term planning}

Day-to-day operation at the mine sites requires a variety of short-term planning, which is facilitated by the DSS/MIS.

The spreadsheet workbook stores the past operational history of the mine in a set of data worksheets, which can be accessed for a variety of analyses.

Operators can call up graphs showing any desired combination of traces (for example, silica content for each pit, as estimated at the mine and reported at the port) for any desired time interval. The data can also be summarized in any chosen manner.

When operational glitches occur, it is helpful to carry out a post-mortem. For example, errant composition values may be ascribed to problems with a particular mine block, or errors relating to the data for a particular train. Capabilities have been incorporated which report the statistics for sources contributing to a particular train; the trains deriving from a particular source, or the sources and trains relating to a chosen time interval. Time interval can be defined either by departure from the mine, or arrival at the port.

\section{Long-term planning}

It is recognized that the DSS described above can reduce only the short-term fluctuations in ore composition, of up to a few days' duration. However, the system provides information invaluable for long-term planning of the mines and for marketing.

The MIS gives early warning if material available from the mine is systematically off-target, enabling timely modification of the monthly and yearly mining and marketing plans.

\section{Conclusion}

The Decision Support System, initially developed for a well-defined and limited purpose, is evolving into a fullyfledged Management Informing System, accessed and used by an ever-widening circle of the company's managers.

Based upon this experience, the developers strongly believe that this incremental and evolutionary approach to developing a Management Informing System is more likely to be successful than is the thoroughly planned, predetermined approach advocated in the textbooks.
The methods described have produced proven increase in product quality with no increase in operating cost. Indeed, operating costs have been reduced to the extent that the control staff can now do their daily planning much more quickly, releasing them to spend more time supervising operations in the mine pits.

As a side benefit to the operational improvements, the methods have provided considerable enhancements in control, reporting, planning and accountability.

\section{References}

Albright, S. C (2001). VBA for Modelers. Pacific Grove, CA: Duxbury.

Australian Bureau of Statistics (2001), Australia Now. Canberra: ABS. Accessed March 29, 2001 at http://www.abs.gov.au

Everett, J.E. (1996). Iron ore handling procedures enhance export quality. Interfaces, 26(6), 82-94.

Everett, J.E. (1997). Simulation to reduce variability in iron ore stockpiles. Mathematics and Computers in Simulation, 43, 563-568.

Everett, J.E. (2001). Iron ore production scheduling to improve product quality. European Journal of Operational Research, 129, 355361.

Everett, J.E., \& Kamperman, M. (1999). A simulation model of iron ore production sequencing to achieve uniform blend. Proceedings of the International Conference on Industrial Logistics, 337-345. St Petersburg, Russia; ICIL '99.

Hanke, J. E., \& Reitsch, A.G. (1998). Business Forecasting. Sixth ed. Englewood Cliffs, NJ: Prentice-Hall.

Taha, H. A (1987). Operations Research. Fourth ed. New York: Macmillan.

TEX (1995). Iron Ore Manual 1994-95. Tokyo: The TEX Report Co.

\section{Biographies}

Jim Everett is Professor of Information Management at the University of Western Australia, within the Faculty of Economics and Commerce. Jim's publications and research interests are outlined on his website http://www.imm.ecel.uwa.edu.au/je.

Terry Howard is Manager, Competitive Improvement Programme, at BHP Iron Ore. Terry is a qualified metallurgist with a Masters Degree in the field of secondary metallurgy. He has 32 years experience in the Australian minerals processing industry, in both primary and secondary processing fields. 


\section{An MIS for Iron Ore Mining Operations}

Marcel Kamperman is Superintendent, Planning, at BHP Iron Ore's facilities in Port Hedland. Marcel is a qualified geologist and chemist with a Ph.D. in the field of mineral chemistry. He has 16 years experience in the Australian minerals industry, in exploration, mining and process control. 